\title{
AGENESIA UNILATERAL DE LA ARTERIA PULMONAR. PRESENTACION DE DOS CASOS Y REVISION DE LA LITERATURA
}

\author{
UNILATERAL AGENESIS PULMONARY ARTERY \\ PRESENTATION OF TWO CASES AND REVIEW OF THE LITERATURE
}

Dr Julio Maggiolo M. ${ }^{1}$, Dra Lilian Rubilar 0. ${ }^{1}$

1.- Pediatra Especialista en Enfermedades Respiratorias. Unidad Broncopulmonar. Hospital Exequiel González Cortés. Santiago, Chile.

\begin{abstract}
Unilateral agenesis of the pulmonary artery is a rare malformation, sometimes asymptomatic and underdiagnosed. Right agenesis is usually isolated, while the left agenesis is more frequently associated with cardiovascular malformations. Some patients have recurrent respiratory infections, exercise limitation, hemoptysis, and / or pulmonary hypertension. The diagnosis is suspected by chest radiograph showing a hypoplastic lung ipsilateral to the agenesis. It is confirmed with contrast-enhanced chest computed tomography. In asymptomatic patients, management should be conservative, however, the search for collateral vessels should be started from adolescence, because they can bleed. In symptomatic patients or those with serious complications, the treatment must be interventional. The objective of this article is to present two patients with right and left pulmonary artery agenesis respectively and review the literature.
\end{abstract}

Key words: agenesis pulmonary artery, malformation, hemoptysis, pulmonary hypoplasia.

\section{RESUMEN}

La agenesia unilateral de la arteria pulmonar es una malformación poco frecuente, en ocasiones asintomática y subdiagnosticada. La agenesia derecha suele ser aislada, en cambio la izquierda se asocia más frecuentemente a malformaciones cardiovasculares. Algunos pacientes presentan infecciones respiratorias recurrentes, limitación al ejercicio, hemoptisis y/o hipertensión pulmonar. El diagnóstico se sospecha al solicitar una radiografía de tórax que muestra un pulmón hipoplásico ipsilateral a la agenesia. Se confirma con tomografía computada de tórax con contraste. En pacientes asintomáticos el manejo debe ser conservador, sin embargo, se debe iniciar la búsqueda de vasos colaterales especialmente durante la adolescencia, los que pueden sangrar. En cambio, en los pacientes sintomáticos o que presentan complicaciones graves, el tratamiento debe ser intervencional. El objetivo de este trabajo es presentar a dos pacientes con agenesia de la arteria pulmonar derecha e izquierda respectivamente y hacer una revisión de la literatura.

Palabras clave: agenesia arteria pulmonar, malformación, hemoptisis, hipoplasia pulmonar.

\section{INTRODUCCIÓN}

La agenesia unilateral de la arteria pulmonar (AUAP) es una entidad poco frecuente. Siendo más prevalente la agenesia de la arteria pulmonar derecha (AAPD), la que habitualmente se presenta en forma aislada, es asintomática y subdiagnosticada. El diagnóstico suele ser un hallazgo al solicitar una radiografía (Rx) de tórax por otros motivos y se confirma mediante una tomografía computada (TC) de tórax con medio de contraste.

En cambio, la agenesia de la arteria pulmonar izquierda (AAPI) se asocia a otras malformaciones cardiovasculares, presentando mayor sintomatología. La tomografía computarizada por emisión de fotón simple (SPECT) evidencia ausencia de perfusión del pulmón afectado.

El tratamiento debe ser conservador en pacientes asintomáticos y más invasivo en aquellos con sintomatología 0 que presenten complicaciones graves.

El pulmón hipoplásico es irrigado por arterias colaterales sistémicas, pudiendo producir sangrados, especial-

\section{Correspondencia:}

Dr. Julio Maggiolo M

maggiolojulio@gmail.com mente en la adolescencia, si es recurrente o masivo, se debe realizar embolización y si es refractario al tratamiento se recurrirá a la lobectomía o neumonectomía.

La Figura 1 muestra la anatomía normal de las arterias pulmonares en imágenes de TC y Rx de tórax.

\section{CASOS CLÍNICOS}

Caso clínico 1.- Varón de 16 años de edad, controlado en la unidad broncopulmonar del Hospital Exequiel González Cortés, en Santiago, Chile. Sin antecedentes mórbidos neonatales, personales, ni familiares.

Desde los 2 meses de edad presentó retracción de partes blandas y desde los 10 meses deformidad torácica. Se solicitó una Rx de tórax y una TC de tórax, cuyas imágenes fueron interpretadas como un enfisema lobar congénito (ELC) del lóbulo superior izquierdo (LSI).

El paciente fue intervenido quirúrgicamente durante el primer año de edad, practicándose lobectomía del LSI. Sin embargo, el resultado de la biopsia fue compatible con hiperinsuflación del LSI.

Durante el período de lactante el paciente evoluciona con cuadros bronquiales obstructivos recurrentes, con 
Figura 1. Anatomía normal de las arterias pulmonares. La arteria pulmonar derecha (marcador en a y b) se localiza anterior e inferior al bronquio principal derecho. La arteria pulmonar izquierda (marcador en c y d) se dispone superior al bronquio principal izquierdo (punta de flecha en d) y es la causante de la posición más alta del hilio pulmonar izquierdo en la radiografía de tórax normal (flecha en e) (8). Con autorización de los autores.
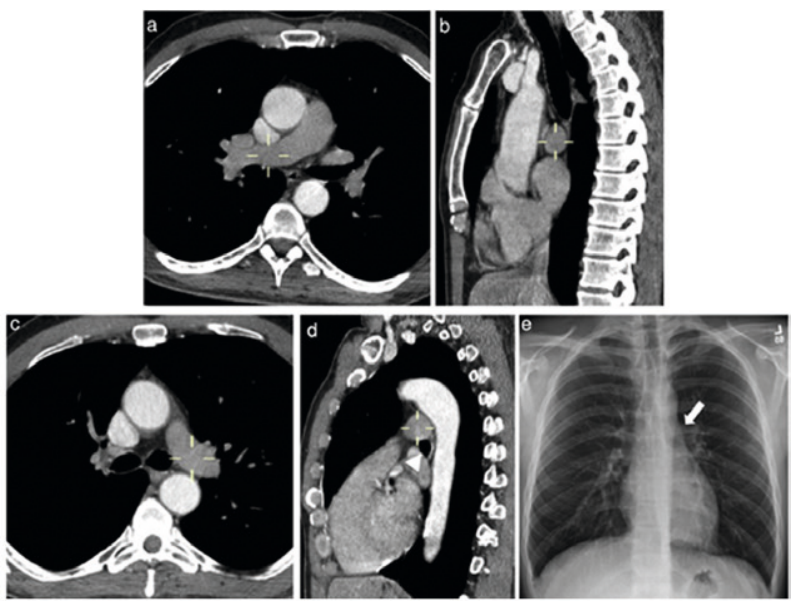

una frecuencia aproximada de 3 a 4 crisis al año. Se indicó salbutamol en las crisis, fluticasona 250 ug/día y prednisona 1 a 2 curas al año. Durante la etapa preescolar disminuyó la frecuencia a 1 crisis por año.

Un test de ejercicio solicitado a los 5 años de edad fué negativo. Las espirometrías realizadas a los 6 años, con equipo Med Graphics y utilizando valores de referencia de Knudson (1), mostraron un patrón ventilatorio persistentemente normal. Sin embargo, la última hecha a los 15 años, utilizando valores de referencia de Gutiérrex (2) para su interpretación, mostró una alteración ventilatoria obstructiva leve con Capacidad Vital Forzada (CVF) normal, FEF25-75 (Flujo espiratorio en el 25 a $75 \%$ de la CVF) disminuido, sin cambios significativos post broncodilatador. Una pletismografía (realizada con equipo Med Graphics), mostró capacidad pulmonar total (CPT) disminuida leve, índice de atrapamiento aéreo aumentado a expensas del volumen residual (VR) y resistencia de la vía aérea aumentada, con cambio significativo post broncodilatador y la capacidad de difusión (DICO) fue normal. Se solicitó un ecocardiograma con Doppler color, que mostró dextroposición cardíaca, descartando malformaciones cardiovasculares e hipertensión pulmonar (HTP).

Debido a la buena evolución broncopulmonar del paciente, no se solicitaron estudios radiológicos durante la edad escolar. En una Rx de tórax practicada a los 13 años de edad, se vio desviación de la tráquea a derecha, imagen repetida en los siguientes controles radiográficos, lo cual no era concordante con la lobectomía de LSI realizada al año de vida.

Se decide solicitar una TC de tórax y TC de abdomen con medio de contraste (Figura 2 y 3), la que muestra hipoplasia del pulmón derecho, agenesia de la arteria pulmonar
Figura 2. TC tórax con medio de contraste (caso clínico 1): hipoplasia pulmonar derecha, e hiperinsuflación compensatoria del pulmón izquierdo. Agenesia de la arteria pulmonar derecha. La flecha muestra la arteria pulmonar izquierda.

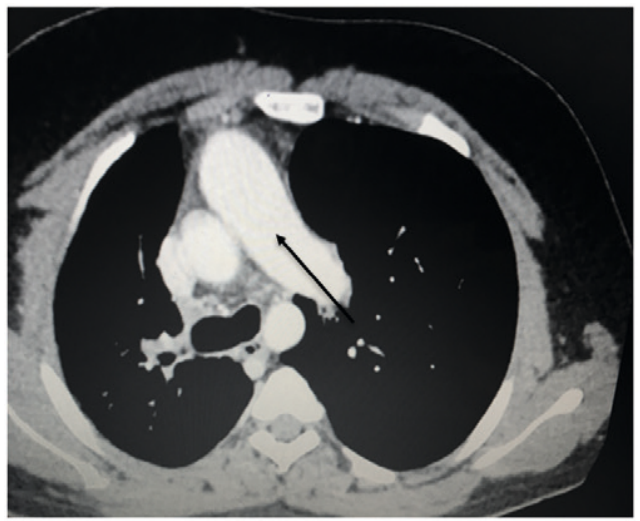

Figura 3. TC de tórax y de abdomen con medio de contraste (caso clínico 1): a: vaso colateral que nace de la aorta abdominal, atraviesa el diafragma y entra al hilio derecho (flecha), b: pequeño vaso colateral que nace lateral a la arteria mesentérica superior y sube hacia al hilio derecho (flecha).
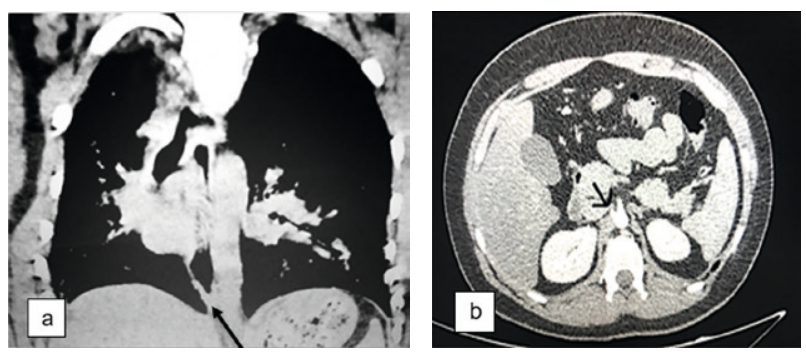

derecha y presencia de arterias sistémicas colaterales que nacen de la aorta. La SPECT de perfusión evidenció ausencia de perfusión del pulmón derecho.

El paciente evolucionó con reagudizaciones obstructivas e infecciones pulmonares recurrentes, tratadas con salbutamol, prednisona y diferentes esquemas antibióticos. A los 16 años presenta un cuadro de hemoptisis, debido a lo cual fue derivado para su hospitalización. Se solicitó angioTC de tórax que evidenció buen llene del tronco de la arteria pulmonar, el medio contraste mostró la rama izquierda hasta niveles subsegmentarios, en cambio la rama derecha se observó estrecha, con lumen irregular, sin medio de contraste hacia distal, además de colaterales que irrigaban al pulmón derecho, procedentes de la aorta abdominal.

El paciente presentó una hemoptisis masiva, por lo que se decidió practicar embolización de la arteria subclavia derecha, sin embargo, por refractariedad a esta terapia se realizó neumonectomía derecha (Figura 4).

Al momento de esta publicación el paciente se encontraba estable. 
Figura 4. Rx de tórax (a) y TC de tórax (b) con medio de contraste (caso clínico 1), post neumonectomía derecha. En ambas imágenes se observa sólo el pulmón izquierdo, desviación del mediastino a derecha e hiperinsuflación compensatoria del pulmón izquierdo.
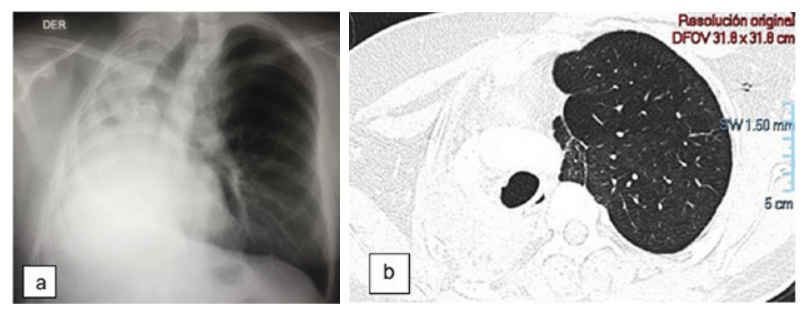

Caso clínico 2.- Varón de 15 años de edad, portador de Tetralogía de Fallot, atresia pulmonar, comunicación interventricular (CIV) subaórtica amplia, dilatación e hipertrofia moderada del ventrículo derecho, vena cava izquierda persisten, sin HTP.

El paciente presenta cianosis, hipocratismo digital, deformidad torácica, limitación para hacer ejercicios de mediana intensidad. Además de 3 a 4 bronquitis obstructivas anuales y broncorrea mucopurulenta persistente, con buena respuesta a diversos esquemas antibióticos. Ha sido hospitalizado en numerosas oportunidades, por descompensaciones cardíacas e infecciones bacterianas broncopulmonares. Presentó hemoptisis leve, que cedió espontáneamente, descartándose vasos colaterales. Fue intervenido quirúrgicamente en repetidas ocasiones, para practicársele cirugías cardíacas paliativas.

Figura 5. Rx tórax (caso clínico 2): hipoplasia pulmonar izquierda, disminución del volumen del pulmón izquierdo (flecha), hiperinsuflación pulmonar derecha, que se observa por aumento de volumen del pulmón derecho, desviación del mediastino a izquierda.

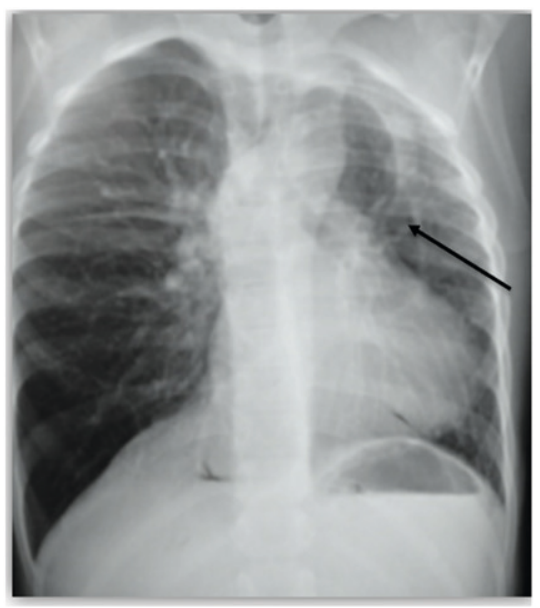

La Rx tórax (Figura 5) evidencia hipoplasia pulmonar izquierda, hiperinsuflación del pulmón derecho, dextroposición cardíaca izquierda. La TC de tórax con contraste (Figura 6) muestra mediastino desviado a izquierda, no se identifica la arteria pulmonar izquierda, sin imágenes de condensación. En la ventana pulmonar se observan bronquiectasias. No colabora para realizar una espirometría aceptable y reproducible.

Al momento de esta publicación, el paciente se encontraba estable, en espera de una intervención quirúrgica definitiva.

Figura 6. TC de tórax con medio de contraste, ventana mediastínica (caso clínico 2): agenesia de la arteria pulmonar izquierda, hipoplasia pulmonar izquierda, se observa disminución del volumen pulmonar izquierdo. La flecha muestra la arteria pulmonar derecha.

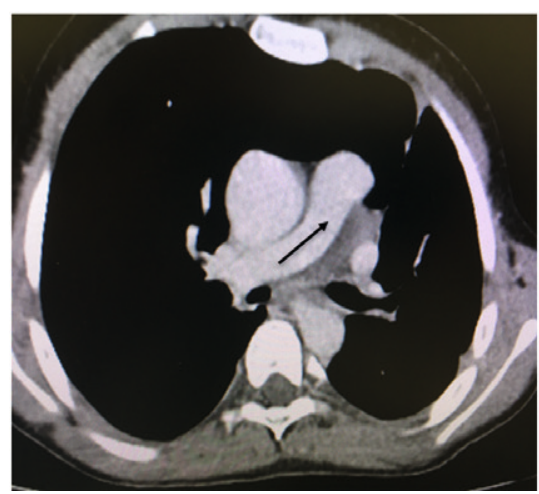

\section{DISCUSION}

La AUAP es una malformación poco frecuente, su prevalencia es de 1 por cada 200.000-300.000 individuos y en la edad pediátrica suele ser subdiagnosticada $(3,4)$.

Se produce como consecuencia de la involución intrauterina del sexto arco aórtico, que condiciona la ausencia de la porción proximal de la arteria pulmonar derecha 0 izquierda ${ }^{(5,6)}$.

El desarrollo pulmonar fetal y su anatomía broncovascular suelen ser normales, debido a que la parte hiliar y distal de la arteria pulmonar y sus ramas están irrigadas por el ductus arterioso ipsilateral, pero sin conexión con el tronco de las arterias pulmonares $(5,6)$. Posteriormente al nacimiento, debido al cierre de dicho conducto, se produce una disminución del flujo sanguíneo, lo que origina hipoplasia pulmonar y desarrollo de colaterales aortopulmonares (7).

Los pacientes con AUAP aislada pueden evolucionar asintomáticos, detectándose inicialmente como un hallazgo casual al realizar una $\mathrm{Rx}$ de tórax, donde se observa en el mismo hemitórax de la agenesia, un pulmón disminuido de tamaño con desplazamiento mediastínico ipsilateral, asociado a hiperinsuflación pulmonar contralateral, con posible herniación hacia el lado afectado (7-9). Esta herniación, con hiperinsuflación compensatoria, Ilevó al diagnóstico erróneo de ELC en nuestro caso clínico 1, al que se le practicó lobectomía LSI.

La AAPD es mucho más frecuente que la izquierda, habitualmente se presenta aislada, observándose menos anormalidades asociadas, pudiendo ser asintomática. La circulación pulmonar usualmente se realiza a través de arterias 
bronquiales hipertróficas, algunos pacientes pueden presentar arco aórtico izquierdo o arteria innominada derecha. En la mayoría de los pacientes existe desarrollo de circulación colateral proveniente de la aorta descendente, especialmente abdominal (7-9). Debido a que estas arterias son friables, si se añade a esto que durante la adolescencia ocurre un crecimiento acelerado, es posible que sangren, lo que podría producir una hemorragia masiva (10), como le ocurrió al paciente del caso clínico 1, al que se le practicó una neumonectomía derecha, debido a la falta de respuesta de la embolización de las arterias colaterales. Es por este motivo que se los vasos colaterales deben ser pesquisados precozmente.

En cambio, en los pacientes portadores de AAPI se observa una alta asociación a malformaciones cardiovasculares, como tetralogía de Fallot, defectos del tabique interauricular o interventricular, como el caso clínico 2, además de transposición de grandes vasos y ductus arterioso persistente (11). Los niños menores de un año de edad pueden presentar distrés respiratorio (56\%) asociados o no a HTP y desarrollo posterior de insuficiencia cardiaca. Luego del año de edad pueden desarrollar infecciones respiratorias recurrentes (23$37 \%$ ), hemoptisis (14-20\%) por la excesiva circulación colateral aortopulmonar (3), lo que también ocurrió con el segundo caso clínico, siendo de menor cuantía que en el caso 1. El flujo sanguíneo del parénquima pulmonar proviene de la aorta descendente, la arteria innominada izquierda, la subclavia 0 por circulación bronquial hipertrófica (7).

El diagnóstico definitivo de la AUAP debe basarse en las pruebas de imágenes $(8,9)$, como ya se mencionó, la Rx tórax demuestra hipoplasia pulmonar ipsilateral a la arteria ausente, debiendo confirmarse con una TC tórax con medio de contraste (8). En la ecocardiografía con Doppler color se pueden detectar malformaciones cardiovasculares asociadas (9), HTP y dextroposición cardíaca. SPECT demuestra exclusión de la perfusión completa del lado comprometido, con ventilación normal (9). La arteriografía pulmonar a pesar de que se utiliza menos, confirma la ausencia de la arteria comprometida, muestra otras anormalidades vasculares asociadas y permite identificar el origen anómalo de las arterias colaterales a partir de la circulación sistémica (9).

La espirometría y los volúmenes pulmonares pueden ser normales o mostrar alteraciones restrictivas leves. La DICO es normal (7).

Si en el seguimiento se pesquisan arterias colaterales, o bien se presentan complicaciones como hemoptisis moderadas, masivas o recurrentes (10), se debe realizar embolización arterial $(12,13)$, si es refractaria a la terapia se recurrirá a la Iobectomía o incluso a la neumonectomía $(12,14)$, como lo que ocurrió con el caso clínico 1.

Las infecciones respiratorias recurrentes se asocian con AUAP, deben ser tratadas en forma enérgica para evitar la aparición de bronquiectasias (7). $(12,15)$.

La HTP se debe tratar con fármacos hipotensores

La cirugía reparadora de elección consta de 2 etapas, anastomosis quirúrgica de la parte proximal y distal de la arteria pulmonar y posterior implantación de stent.
$(12,14,15)$. Algunos autores la realizan aún en casos asintomáticos, pero su indicación es muy controvertida, debido a las altas probabilidades de reintervención por complicaciones posquirúrgicas, como la trombosis de la anastomosis $(14,15)$.

\section{CONCLUSIONES}

La AUAP es una patología poco frecuente, la AUAPD se debe sospechar en pacientes que a pesar de ser asintomático u oligosintomáticos, la Rx de tórax muestra desviación del mediastino hacia el pulmón que presenta la agenesia, por hipoplasia pulmonar, la TC de tórax con medio de contraste muestra ausencia unilateral de la arteria pulmonar derecha. Es importante buscar la presencia de arterias colaterales, las que habitualmente se originan en la aorta, las cuales especialmente en la adolescencia pueden sangrar y presentar hemoptisis significativa, la que requerirá embolización y si resulta refractaria a esta terapia, se debe recurrir a la neumonectomía.

La AUAPI presenta más síntomas debido a que se asocia a otras malformaciones cardiovasculares, una vez diagnosticada, también se deben buscar arterias colaterales.

Por último, en general, en los pacientes asintomáticos el tratamiento debe ser conservador, en cambio debe ser invasivo en aquellos con sintomatología o que presenten complicaciones graves.

\section{Revisores de este artículo}

Dr. Jury Hernández C.

Pediatra Broncopulmonar

Complejo Asistencial Dr. Víctor Ríos Ruiz

Los Ángeles, Biobío. Chile.

Dr. Pablo Bertrand N.

Pediatra Broncopulmonar

Académico, Escuela de Medicina,

Pontificia Universidad Católica de Chile.

Santiago, Chile.

Fecha de recepción: junio 2020

Fecha de publicación: marzo 2021

\section{REFERENCIAS BIBLIOGRAFICAS}

1. Knudson RJ, Lebowitz MD, Holberg Cl, Burrows B. Changes in the normal maximal expiratory flow-volume with growth and aging. Am Rev Respir Dis 1983; 127: 72734.

2. Gutiérrez M, Rioseco F, Rojas A, Casanova D. Ecuaciones de referencia espirométrica en población chilena. Rev Chil Enf Respir 1997; 13: 165-77.

3. Koga T, Hidaka K, Miyako N, Suga N, Takahashi $H$. Age-related clinical characteristics of isolated congenital unilateral absence of a pulmonary artery. Pediatr Cardiol 
2010; 31: 1186-90. http://dx.doi.org/10.1007/s00246010-9787-5

4. David W, Reading R, Umesh P, Oza C. Unilateral absence of a pulmonary artery: a rare disorder with variable presentation. Proc (Bayl Univ Med Cent) 2012; 25(2): 115-18.

5. Lagman J. Sistema cardiovascular. En Embriología Médica. $3^{\circ}$ edición. Interamericana, 1976.

6. Davies M, Guest PJ. Developmental abnormalities of the great vessels of the thorax and their embryological basis. Br J Radiol 2003; 76: 491-502.

7. Kruzliak P, Syamasundar RP, Novak M, Pechanova 0 , Kovacova G. Unilateral absence of pulmonary artery: pathophysiology, symptoms, diagnosis and current treatment. Arch Cardiovasc Dis 2013; 106(8-9): 448-54. http://dx.doi.org/10.1016/j.acvd.2013.05.004

8. Bueno J, Flors L, Mejía M. Anomalías congénitas de las arterias pulmonares: espectro de hallazgos en tomografía computarizada. Radiología 2017; 59 (3): 209-1. http://dx.doi.org/10.1016/j.rx.2016.09.0080033-8338

9. Yiu M, Le D, Leung $Y$, Ooi C. Radiological features of isolated unilateral absence of the pulmonary artery. J HK Coll Radiol 2001; 4: 277-80

10. Thomas P, Reynaud- Gaubert M, Bartoli JM. Exsanguinating Hemoptysis Revealing the Absence of Left Pulmonary Artery. Ann Thorac Surg 2001; 72(5): 1748-50.
11. Lozano Rodríguez A, Junquera Rionda P, Santana Montesdeoca JM, Fornell Pérez R. Solution to case 33. Unilateral proximal interruption of the left pulmonary artery. Radiología 2011; 53(5): 476-9.

12. Krammoh J.L, Bigras M, Prsa C, Lapierre J, Miró N.S, Dahdah N. Therapeutic strategies in children with an isolated unilaterally absent proximal pulmonary artery. Cardiol 2010; 31: 607-10. http://dx.doi.org/10.1007/ s00246-010-9689-6

13. Rene M, Sans J. Unilateral pulmonary artery agenesis presenting with hemoptysis: treatment by embolization of systemic collaterals. Cardiovasc Intervent. Radiol 1995; 18: $251-4$.

14. Alison M, Garel L, Bigras JL, Déry J, Lapierre C. Unilateral absence of pulmonary artery in children: Bronchovascular anatomy, natural course and effect of treatment on lung growth. Pediatr Radiol 2011; 41: 459-68. http:// dx.doi.org/10.1007/s00247-010-1877-2

15. Umezu K, Harada Y, Sakamoto T, Takigiku K, Yasukochi $S$. Ausencia unilateral de la arteria pulmonar proximal derecha en el contexto de hipertensión pulmonar: consideraciones para la reparación quirúrgica y el tratamiento perioperatorio. Gen Thorac Cardiovasc Surg 2012; 60 (6): $367-9$. 